\title{
Jeux et enjeux de l’Union Européenne : quels enseignements peut-on tirer?
}

\author{
Liridon Lika ${ }^{1}$ et Audrey Weerts ${ }^{2}$
}

Mots-clés: Union Européenne ; politique étrangère ; politique commerciale ; atouts ; défis ; enjeux

Les contributions de ce numéro spécial ont confirmé l'intérêt qui existe autour du projet européen. Depuis les premières étapes de la construction européenne dans les années 1950, l'Union Européenne (UE) a bien évolué. Partagée entre mouvements d'approfondissement et d'élargissement, elle reste toujours omniprésente dans les débats scientifiques.

Le présent numéro avait précisément pour objectif d'analyser les atouts dont dispose l'UE dans un contexte où les défis et les enjeux sont nombreux pour l'Organisation, tant dans une dimension interne qu'externe. Si l'UE présente de nombreux atouts majeurs en matière de pacification, de réconciliation, de prospérité ou encore de rapprochement entre les populations, les défis et les enjeux pour les instances européennes restent multiples et importants. La question de l'élargissement, celle du Brexit, la crise migratoire, la montée des extrémismes, la pandémie du coronavirus, l'action extérieure, les relations avec le reste du monde, ou encore les réformes institutionnelles animent les débats au sein de l'UE. Dans un tel contexte, de quelles forces dispose l'UE pour mener ces projets ? À l'opposé, quels facteurs la déstabilisent ?

Deux angles ont été particulièrement ciblés dans les contributions, le premier concerne la politique étrangère et de sécurité, le second la politique commerciale de l'UE. Après une présentation générale de Sebastian Santander, les contributions d'André Dumoulin et de Kamal Bayramzadeh se sont interrogées sur les domaines de défense et de sécurité. Quant à celles de Philippe Vincent et de Vincent Bricart, elles se sont focalisées sur la politique commerciale.

\section{L'UE sous différents angles : l'apport des différentes contributions}

Comme nous l'a rappelé Sebastian Santander dans son article, l'UE est une entité au dessein ambitieux et évolutif. Une fois les premiers objectifs atteints (contrer le déclin de l'Europe et pacifier le continent), l'UE a sans cesse travailler à développer et consolider son projet et sa structure. Dans ce cheminement, il y a eu évidemment des obstacles, des défis et même des crises, qui ont aussi contribué à façonner l'entité que nous connaissons aujourd'hui. L'UE, avec ses atouts et ses faiblesses, a toujours suscité l'intérêt des chercheurs. Initialement cependant, la dimension interne au projet était privilégiée par les chercheurs. Néanmoins, à mesure que le projet européen s'est développé et étoffé, l'intérêt pour la dimension extérieure

\footnotetext{
${ }^{1}$ Liridon Lika est chargé de cours adjoint et maître de conférences au Center for International Relations Studies (CEFIR) du Département de Science politique de l'Université de Liège (ULiège). Il est également chercheur postdoctoral à la Faculty of Arts and Social Sciences FASoS) de l'Université de Maastricht (UM). Liridon Lika est titulaire d'une thèse de doctorat en sciences politiques et sociales de l'ULiège (2020).

${ }^{2}$ Audrey Weerts est assistante au Département de Science politique de l'Université de Liège (ULiège). À la fois membre de l'unité d'étude des systèmes politiques belges et de la Cellule d'Appui Politologique Afrique-Caraïbes (CAPAC-ULiège), elle est titulaire d'un master en relations internationales (ULiège) et d'un master en sciences de gestion (HEC-ULiège).
} 
du projet est allé grandissant. Les contributions de ce numéro le démontrent très bien et vont nous permettre de mieux appréhender cette entité sui generis et son activité extérieure.

Dans son article André Dumoulin a analysé la flexibilité conditionnelle dans le domaine de la sécurité coopérative et de la défense au sein de l'UE qui renvoie à l'idée de volontariat et de souveraineté des États. Il a mis en exergue que, dans le domaine de la sécurité-défense, les États membres sont les maîtres du jeu. Dès lors, il souligne que l'aboutissement d'une véritable autonomie diplomatique européenne, parallèlement à une souveraineté européenne partagée, est dépendant d'une analyse commune des menaces, de la définition des intérêts et des capacités, et notamment de la cohérence des États membres dans le domaine de la politique étrangère. L'auteur montre que, bien que l'UE pèse $22 \%$ de l'économie mondiale, elle ne dispose pas encore d'une analyse vraiment partagée de la menace aboutissant à une vision stratégique commune, impliquant un territoire à défendre et une puissance à affirmer face aux nouveaux défis mondiaux. Pour l'auteur, l'intégration politico-militaire est lente et reste fragile mais elle devrait aboutir plus rapidement si le paysage européen devait être concrètement menacé dans un environnement qui, aujourd'hui, voit réapparaitre la compétition militaire parallèlement à la compétition commerciale et technologique et des rivalités de puissance. Dans ce cas-ci, l'idée d'aller vers une plus grande intégration politico-militaire est mise en avant. De plus, l'auteur souligne que l'avenir de la Politique de Sécurité et de Défense Commune (PSDC) sera dépendant aussi du paysage extrêmement mouvant d'aujourd'hui : Brexit, place de la Turquie dans le champ sécuritaire euro-atlantique, positionnement du curseur transatlantique et américain en Europe, perception sécuritaire des mouvements migratoires et le niveau d'implication de la PSDC dans ce cadre, ou encore l'évolution des relations franco-allemandes.

Kamal Bayramzadeh a proposé une contribution avec pour objectif de montrer la limite de la puissance de l'UE dans les relations internationales. Il s'est notamment concentré sur l'exemple du projet nucléaire iranien de 2015 afin d'analyser les divergences entre les ÉtatsUnis et l'UE sur ce sujet, plus particulièrement depuis l'arrivée au pouvoir de Donald Trump. Afin d'étudier cette thématique, il a mobilisé plusieurs concepts des relations internationales tels que celui d'acteur, de puissance douce (soft power) et de puissance normative. Dans cette contribution l'auteur a montré que depuis 1991, l'UE a l'ambition de devenir un acteur important des relations internationales, en participant à la médiation, à la gestion des crises, à l'instauration de la paix et à la démocratisation de plusieurs pays. Cependant, il a souligné également que son rôle doit être mesuré en fonction du contexte, des conditions politiques et du facteur des rapports des forces. Étant donné que l'UE est une organisation régionale, elle ne peut pas être comparée à la puissance d'un État. D'après l'auteur, bien que la puissance normative de l'UE trouve un écho favorable dans ses relations internationales en particulier parmi les pays qui sont candidats ou candidats potentiels à l'adhésion dans l'UE, tels que ceux des Balkans occidentaux, elle connaît des limites dans d'autres pays comme la Russie et la Chine qui n'acceptent pas les normes européennes relatives à la question des droits de l'homme. Pour ce qui est de la limite de la puissance normative de l'UE à l'égard de l'Iran, il l'a examiné à travers quatre périodes de leurs relations : 1) de 1992 à 1997, 2) de 1998 à 2005, 3) de 2005 à 2013 et 4) de 2013 à 2020.

Philippe Vincent s'est ensuite intéressé aux nouveaux accords commerciaux conclus par l'UE ces dernières années. Il dresse le constat, qu'à nos jours, le cadre des relations commerciales de l'UE a énormément changé par rapport à celui qui était en vigueur en 1994 au moment de l'officialisation de l'Organisation Mondiale du Commerce (OMC). Dans son développement, il souligne que l'échec du multilatéralisme commercial a incité la plupart des États du monde, tant émergents qu'industrialisés, a adopté leurs stratégies bilatérales. Ainsi, les puissances émergentes comme la Chine et l'Inde se sont lancées dans le bilatéralisme, négociant des accords avec de nombreux pays du monde. En même temps, souligne l'auteur, les ÉtatsUnis ont, depuis l'arrivée au pouvoir de Donald Trump, fait retour en arrière sur la plupart des 
traités bilatéraux ou multilatéraux restreints qu'ils étaient en train de négocier et n'hésitent pas à appliquer des surcharges tarifaires unilatérales lorsqu'ils estiment que leurs partenaires commerciaux, plus particulièrement l'UE, ne respectent pas les règles du jeu commercial international. Quant à l'UE, il précise qu'elle multiplie les accords, même si ceux-ci se heurtent à l'opposition d'une partie de l'opinion publique, qui est critique et perçoit ces accords comme des simples instruments au service des multinationales visant à réaliser des profits et dans certains cas échappant à l'ordre juridique national des États hôtes. L'auteur s'est également penché sur une brève analyse du contenu des accords, touchant les aspects du commerce des marchandises, du commerce des services, de la propriété intellectuelle, des marchés publics, ou encore sur la question de la protection des investissements.

Toujours en matière de politique commerciale, Vincent Bricart s'est quant à lui penché sur le rôle des assemblées parlementaires dans la politique commerciale extérieure des ÉtatsUnis et de l'UE. Il a apporté une réponse positive à l'analyse de l'existence d'une influence claire des parlementaires européens et américains sur la diplomatie menée par les États-Unis et l'UE, dans la conclusion des traités commerciaux internationaux. Il a démontré que l'influence se manifeste de nombreuses manières telles que, entre autres, le contrôle, l'évaluation et la supervision des négociations par les parlementaires, la création du débat par les institutions législatives en définissant leurs priorités et objectifs, remettant en cause certains points ou décisions des traités négociés, élaborant des demandes et discutant avec l'exécutif afin de se faire entendre par ce dernier. Ainsi, l'auteur constate que ces pressions formelles et informelles, venant des pouvoirs législatifs, mais surtout de la société civile, incitent l'exécutif à prendre en considération les revendications des parlementaires. Pour illustrer ses propos, l'auteur évoque les exemples de l'augmentation de la transparence des négociations du Partenariat Transatlantique de Commerce et d'Investissement ou en anglais Transatlantic Trade and Investment Partnership (TTIP) et de l'Accord Économique et Commercial Global ou en anglais Comprehensive Economic and Trade Agreement (CETA) dans le cas du Parlement européen, d'un côté, et de la définition des objectifs de négociations du TTIP par le Congrès, de l'autre. Néanmoins, l'auteur émet également des réserves à cette influence, puisque l'implication de l'ensemble des assemblées parlementaires des pays démocratiques et à l'ensemble des accords commerciaux n'est pas systématique à chaque État et sur chaque sujet.

\section{Politique étrangère et politique commerciale : quels atouts, quels défis ?}

D'un point de vue externe, l'UE s'est imposée comme un modèle de référence en matière d'intégration régionale et son pouvoir d'attraction reste considérable. Elle exerce une présence solide et une activité multidimensionnelle sur la scène internationale. Cependant, elle peine encore parfois à s'exprimer d'une seule voix et à développer une politique étrangère commune. De ce fait, plusieurs recherches ont abordé la question de la qualification de l'UE comme acteur international à part entière ${ }^{1}$. Dans un contexte de décentrage du pouvoir mondial, quelle peut être la place occupée par l'UE ? Quels sont les atouts dont elle dispose pour ce faire ? Quelles relations entretient-elle avec les grandes puissances, les puissances émergentes ou encore les autres organisations régionales?

Les contributions de ce numéro nous permettent d'apporter quelques éclairages à ces interrogations. Deux angles particuliers ont été traités par les auteurs : celui de la politique étrangère et de la politique commerciale.

\footnotetext{
${ }^{1}$ MERLE, M. (1988), Sociologie des relations internationales, Paris, Dalloz, 4 e édition, p. 317 ; JUPILLE, J. and CAPORASO, J. A. (1998), « States, Agency, and Rules: The European Union in Global Environmental Politics », in RHODES, C. (dir.), The European Union in the World Community, Boulder, Lynne Rienner, p. 214; BRETHERTON, C. and VOGLER, J. (2006), The European Union as a Global Actor, London and New York, Routledge; HILL, C., SMITH, M., and VANHOONACKER, S. (2017), International Relations and the European Union, Oxford, Oxford University Press; PETITEVILLE, F. (2002), « L’Union européenne, acteur international 'global' ? Un agenda de recherche », Revue internationale et stratégique, ${ }^{\circ}$ 47, p. 156.
} 


\section{Politique étrangère}

C'est avec le traité de Maastricht signé en 1992 que l'UE s'est dotée d'une politique étrangère et de sécurité commune plus connue sous le sigle de PESC. Si les États membres conservent bien leur souveraineté en matière de politique étrangère, la PESC offre à ces derniers la possibilité de parler d'une seule voix lorsque les intérêts communs le permettent en leur donnant des moyens pour agir ensemble en la matière. Dans ce cadre, l'UE s'est dotée aussi de la PSDC (anciennement Politique Européenne de Sécurité et de Défense, PESD). Avec ces outils, l'UE ambitionne donc de jouer un rôle dans les relations internationales. Mais qu'en estil dans la pratique?

La contribution d'André Dumoulin nous a confirmé que, pour la PSDC, c'est la dimension intergouvernementale qui est bien prédominante. L'UE n'est pas un État, et de ce fait n'a pas de base accès à des compétences régaliennes. Conservant leur souveraineté, les États membres peuvent toujours, pour des motifs divers, refuser de s'engager. S'il existe bien des mécanismes communs, ceux-ci restent limités. L'intégration politico-militaire se fait donc lentement et surtout elle reste fragile. Ce faisant, l'action européenne dans le domaine de la high politics reste limitée. Toutefois, même si elle ne dispose pas de la force, l'UE peut compter sur d'autres atouts pour peser comme puissance sur la scène internationale, à savoir les normes et le commerce ${ }^{1}$. Comme nous l'a montré la contribution de Kamal Bayramzadeh, il semble que ce soit plutôt sous l'angle des normes que l'UE compte exercer sa puissance. L'UE s'appuierait ainsi sur sa capacité à produire des normes (qui discipline et tend à rendre prévisible les actions des autres acteurs). Certaines normes européennes dépassent aujourd'hui le cadre de l'UE, étendant de la sorte la capacité d'influence par exemple sur les six États des Balkans occidentaux (Albanie, Bosnie-Herzégovine, Kosovo, Macédoine du Nord, Monténégro, Serbie) qui visent à adhérer dans le projet commun européen ${ }^{2}$. L'étude proposée par Kamal Bayramzadeh, concernant le cas de l'Iran, a cependant montré que cette politique donnait lieu à des résultats variables selon le contexte, les conditions politiques et les rapports de force et qu'il fallait donc aussi parfois relativiser la puissance normative de l'UE.

\section{Politique commerciale}

Le commerce a toujours été au cœur du projet européen, en prenant une place grandissante jusqu'à aujourd'hui. Actuellement, au niveau mondial, l'UE constitue un grand marché industrialisé. En 2019, le Fonds Monétaire International (FMI), sur base du Produit Intérieur Brut (PIB) montrait que l'UE représente la deuxième puissance économique mondiale après les États-Unis.

En matière de politique commerciale, Philippe Vincent et Vincent Bricart dressent un constat identique : celui de la multiplication de vastes accords commerciaux par l'UE, mais aussi une façon pour l'UE d'imposer ses normes à ses partenaires. Ce changement de cadre commercial pour l'Union s'explique par l'échec du multilatéralisme commercial. Le blocage des négociations dans le cadre de l'OMC ouvre donc la porte à cette " nouvelle génération d'accords ». Si ce changement de paradigme permet à l'UE de rester dans la course face à d'autres acteurs puissants sur la scène internationale, cela suscite aussi des oppositions : manque de transparence, citoyens/consommateurs délaissés pour le profit, risques économiques ou encore risques pour la santé.

\footnotetext{
${ }^{1}$ MANNERS, I. (2002), « Normative Power Europe: A Contradiction in Terms? », Journal of Common Market Studies, vol. 40, n 2, p. 252; LAÏDI, Z. (2009), « L'Europe, puissance normative internationale », in DEHOUSSE R. (dir.), Politiques européennes, Paris, Presses de Sciences Po, pp. 227-242.

${ }^{2}$ LIKA L. (2020), Les Balkans occidentaux à la croisée de l'intégration européenne et de la projection des puissances émergentes. Analyse du cadre triangulaire entre les Balkans occidentaux, l'Union européenne et la Turquie, Thèse de doctorat en science politique et sociale, Université de Liège, mai 2020, pp. 159-164.
} 
Pour compléter cet aperçu sur la politique commerciale, Vincent Bricart ajoute un autre constat, à savoir celui de l'implication croissante des assemblées parlementaires dans le processus de négociation/conclusion des traités commerciaux internationaux : un facteur qui a toute son importance dans le contexte des oppositions à la politique commerciale de l'UE, car cette inclusion du Parlement permet aux dirigeants de l'UE d'apporter une légitimité nouvelle à sa politique.

\section{L'UE à la recherche d'un nouveau narratif mobilisateur : combiner la norme et la force ?}

La variété des articles présentés dans ce numéro spécial nous a permis de conclure que, bien que l'UE soit analysée sous différents angles, elle présente néanmoins de nombreux traits communs.

Depuis 2008, suite à la crise économique et financière, le projet européen a connu une importante crise. D'autres crises peuvent être ajoutées à la liste, comme celles des réfugiés, des conflits géopolitiques autour de l'UE, les populismes, le Brexit, ou encore la plus récente la pandémie du coronavirus. Par exemple, les crises migratoires et du coronavirus ont démontré un certain manque de solidarité entre les États membres qui n'arrivent pas à parler d'une seule voix. Autrement dit, celles-ci ont mis en lumière un manque de cohérence en matière de politique étrangère, voire une absence de vision stratégique commune. Ainsi, certains soulignent que la crise européenne est à la fois une crise d'identité, de fonctionnement, de projet et de finalité ${ }^{1}$. Pour ces derniers, ce qui manque à l'UE c'est un narratif mobilisateur, c'est-àdire l'histoire d'un projet collectif sur l'utilité de l'UE au $\mathrm{XXI}^{\mathrm{e}}$ siècle ; autrement dit, soulignent-ils, l'UE a besoin d'un nouveau contrat basé sur la combinaison de la norme et de la force ${ }^{2}$.

Parmi les atouts clés de l'UE restent sa puissance économique et son pouvoir d'attraction. Comme déjà indiqué supra, sur le plan économique, d'après le FMI et sur base du PIB en 2019, les États-Unis occupent la première place tandis que l'UE représente la deuxième puissance mondiale. Ainsi, dans l'action extérieure de l'UE le commerce est aussi au service de la norme. Sur le plan politique, l'UE exerce un poids de soft power conséquent à l'extérieur. Les Balkans occidentaux constituent un exemple illustratif. L'adhésion à l'UE pour des États comme l'Albanie, le Kosovo, le Monténégro ou encore la Macédoine du Nord est une priorité stratégique de leur gouvernements respectifs ainsi que la volonté d'une très importante partie de leurs citoyens.

\section{Bibliographie}

BRETHERTON, CHARLOTTE and VOGLER, JOHN (2006), The European Union as a Global Actor, London and New York, Routledge.

HILL, CHRISTOPHER, SMITH, MICHAEL and VANHOONACKER, SOPHIE (2017), International Relations and the European Union, Oxford, Oxford University Press.

JUPILLE, JOSEPH and CAPORASO, JAMES A. (1998), "States, Agency, and Rules: The European Union in Global Environmental Politics », in RHODES CAROLYN (dir.), The European Union in the World Community, Boulder, Lynne Rienner.

\footnotetext{
${ }^{1}$ LAMY, P. et GNESSOTO, N. avec BAER, J-M. (2017), Où va le monde? Le marché ou la force?, Paris, Odile Jacob., pp. 175-176.

${ }^{2}$ Ibid., pp. 169-227.
} 
LAÏDI, Z. (2009), « L'Europe, puissance normative internationale », in DEHOUSSE R. (dir.), Politiques européennes, Paris, Presses de Sciences Po, pp. 227-242.

LAMY, P. et GNESSOTO, N. avec BAER, J-M. (2017), Où va le monde? Le marché ou la force?, Paris, Odile Jacob.

LIKA L. (2020), Les Balkans occidentaux à la croisée de l'intégration européenne et de la projection des puissances émergentes. Analyse du cadre triangulaire entre les Balkans occidentaux, l'Union européenne et la Turquie, Thèse de doctorat en science politique et sociale, Université de Liège, mai 2020, 472 p.

MANNERS, I. (2002), « Normative Power Europe: A Contradiction in Terms? », Journal of Common Market Studies, vol. 40, $\mathrm{n}^{\circ}$ 2, pp. 235-258.

MERLE, M. (1988), Sociologie des relations internationales, Paris, Dalloz, $4^{\mathrm{e}}$ édition.

PETITEVILLE, F. (2002), « L’Union européenne, acteur international 'global' ? Un agenda de recherche », Revue internationale et stratégique, $\mathrm{n}^{\circ} 47$, pp. 145-157. 